\title{
Hamiltonian form for general autonomous ODE systems: Introductory results
}

\author{
Artur Kobus*, \\ Uniwersytet w Białymstoku, Wydział Fizyki \\ ul. Ciołkowskiego 1L, 15-245 Białystok, Poland
}

\begin{abstract}
New class of conserved quantities is constructed. These quantities find direct application in mechanics of dissipative (generally non-conservative) dynamical systems. Approach demands formulation in the language of geometric mechanics, providing theoretical framework for situations with energy flow in and out of the system. As a by product, we suggest possibility of existence of Hamiltonian form for every autonomous ODE system, evolution of which is governed by non-potential generator of motion. Various examples are provided, ranging from physics and mathematics, to chemical kinetics and population dynamics in biology. Applications of these ideas in geometric integration techniques (GNI) of numerical analysis are discussed, and as an example of such, new discrete gradient-based numerical method is introduced.
\end{abstract}

MSC 2010: 37N05; 70H33; 34A34; $53 \mathrm{Z} 05$.

Keywords: classical dynamics, conservation laws, ordinary differential equations, phase-space geometry.

\section{Declarations}

Funding Not applicable

Conflicts of interest/Competing interests Author claim no conflicts of interest connected to presented research.

Availability of data and material Not applicable

Code availability Not applicable

\section{Introduction}

Physics clearly distinguishes systems with conservation laws obeyed, as this simplifies the treatment of physical input given by initial or boundary conditions and formal description in terms of admissible mathematical tools. Hamiltonian systems, which are model examples of how conserved quantities fit into physics, are very convenient since their nice formal features and associated structure provided by symplectic geometry additionally reveals several hidden links between objects

\footnotetext{
*E-mail: arturk90fk@gmail.com
} 
engaged in the theory [2]. Nonetheless, often our analytic methods are not sufficient, as we need to ponder the possibility of numerical solution of the problem under consideration..

Numerical integration algorithms range from general-purpose classic Runge-Kutta and multistep schemes [36] to more specific, geometric methods [18]. The latter admit of course better qualitative behavior (e.g. long time stability [11]), while the former usually are of higher order with less flexible adaptation possibilities when it comes to change of simulation circumstances (e.g. scheme fails to maintain high accuracy when simulation lasts too long). Hence geometric methods show some superiority, when we go to extremes of bending simulations parameters.

We find main motivation of the undertaken research in geometric numerical treatment of ODEs (Geometric Numerical Integration, or GNI for short, see e.g. [5, 9, 10, 31]), although this time we confine ourselves to construction of proper continuous counterpart of the needed framework.

As is well-known, geometric methods demand some kind of in-built qualitative structure of the considered problem to be preserved by the scheme. Good example of such structure, and maybe the simplest one, is a single conserved quantity. This case can be reduced to usage of certain discrete gradient algorithm, see e.g. [22, 29, 34]. Such numerical treatment was indeed applied to systems with first integrals and Lyapunov functions, but for arbitrary systems it generally ceased to function properly because of lack of structural property guiding the evolution of the system. Although several frameworks of interest have been proposed for qualitative description of such problems (see e.g. $[15,20,27,35]$ ), none of these provides simple and immediate way to gain access to conserved quantity helping directly in performance of specially crafted numerical algorithm.

In this paper we show that Hamiltonian systems are indeed ubiquitous when we consider some consequences of theorem on existence and uniqueness of solutions to ordinary differential equations (ODEs) $[19,21,26]$. This approach leads to abundance of so-called effectively conserved quantities even in normally-understood non-conservative systems, although appearance of these has different formal aftermath, when compared to traditional first integrals.

Rest of this section settles the ground for further discussion.. Sections 2,3 are clearly of introductory character giving some basic facts on the topic of geometric Hamiltonian mechanics and relatively subjective point of view on $\mathcal{M}$-systems. Section 4 presents framework of this paper with rudimentary explanation and physical interpretation of our method, where also new conserved quantities are found; accompanying examples are given in sec. 5. Final, 6-th section is devoted to conclusions and proposition of new numerical method.

To begin with, let us consider simple IVP (initial value problem) for autonomous ODE

$$
\dot{\boldsymbol{x}}=\boldsymbol{f}(\boldsymbol{x}), \quad \boldsymbol{x}\left(t_{0}\right)=\boldsymbol{x}_{0},
$$

given on some open domain $D \subset \mathbb{R}^{d}$ with $f: D \rightarrow \mathbb{R}^{m}$; obviously an overset dot stands for a shortcoming denoting time derivation.

Given $\boldsymbol{f}(\boldsymbol{x}) \in \mathcal{C}^{0}(D)$, existence and continuity of solutions are guaranteed. Assumption of $\boldsymbol{f}(\boldsymbol{x}) \in \mathcal{C}^{r}(D), r \geq 1$ ensures uniqueness of the solution and its respective differentiability properties $[2,19,26]$.

McLachlan et al. $[29,30]$ have shown that, in a neighborhood of non-degenerate fixed point of a dynamical system, existence of first integral of (1.1) is equivalent with existence and boundedness of skew-symmetric matrix $B(\boldsymbol{x})$ such that

$$
\boldsymbol{f}(\boldsymbol{x})=B(\boldsymbol{x}) \nabla H(\boldsymbol{x}),
$$

where $H(\boldsymbol{x})$ denotes the mentioned integral. Note that $B$ is not determined uniquely, since we can 
add any solution of homogeneous equation $0=B \nabla H$ to particular solution

$$
S^{(P)}=\frac{1}{|\nabla H|^{2}} f \wedge \nabla H
$$

provided by Quispel and Capel in [34]. This explicitly demands $|\nabla H| \neq 0$. Note that if we are already given the problem in a form (1.2), the assumption of $\nabla H \neq 0$ is redundant.

This article is meant to serve as a continuation of unifying gradient systems that has origin in [30], however, we use existence and uniqueness theorem for solutions of ODEs allowing in this way treatment of more general, non-potential systems. although approach dedicated to non-autonomous ones is still in development.

\section{Hamiltonian mechanics}

If $B(\boldsymbol{x})$ in (1.2) obeys the Jacobi identity, we can term the system Poisson or Hamiltonian (noncanonical). Since Poisson structure matrix $B$ is always of an even rank [39], odd dimensional systems are necessarily degenerate (some even-dimensional ones also are; this implies existence of Casimir functions) [23, 40].

If we face degenerate case, we pick constant values for Casimirs and introduce Clebsh variables on symplectic leaves (Casimir level sets - submanifolds with fixed values of Casimirs, possessing non-degenerate Poisson structure) of initial Poisson manifold. Since that, we can always confine ourselves to non-degenerate Poisson structure, through deliberate change of coordinates leading to canonical Hamiltonian system with Darboux coordinates [2, 3].

These coordinates on $T^{*} M$, together with real function $H: T^{*} M \rightarrow \mathbb{R}, H \in \mathcal{C}^{2}(D)$ (at least) gives raise to a Hamiltonian system.

On the chosen leaf treated as symplectic manifold $\left(T^{*} M, \omega\right)$ (formally $\mathrm{M}$ is the configuration space of the system) in canonical coordinates, we have

$$
\omega=d x^{i} \wedge d p_{i}
$$

existence of which is equivalent with non-degenerate canonical Poisson bracket given by Poisson bi-vector

$$
\pi=-\omega^{-1}: \bigwedge^{2} T^{*} M \rightarrow \mathbb{R}
$$

where

$$
\pi(d f, d g)=\{f, g\}
$$

and $f, g \in \mathcal{F}\left(T^{*} M\right)$ Lie algebra of functions.

This also gives rise to first order differential operator

$$
X_{H}(\cdot)=\pi(\cdot, d H)
$$

known as a Hamiltonian vector field.

Now, we call the first integral generator of motion if

$$
\dot{\boldsymbol{x}}=\{\boldsymbol{x}, H\}=X_{H}(\boldsymbol{x})
$$


understood component-wise. In other words

$$
\begin{aligned}
& \dot{x}^{i}=\frac{\partial H}{\partial p_{i}}, \\
& \dot{p}_{i}=-\frac{\partial H}{\partial x^{i}} .
\end{aligned}
$$

or simply

$$
\left.X_{H}\right\rfloor \omega=d H
$$

denoting by $\rfloor$ the substitution of a vector field into a form (contraction).

Note that the flow of Hamiltonian vector field preserves canonical symplectic form on the phasespace, which is clearly given by proper Lie-Ślebodziński derivative

$$
\left.\left.\mathcal{L}_{X_{H}}(\omega)=X_{H}\right\rfloor d \omega+d\left(X_{H}\right\rfloor \omega\right)=d(d H)=0,
$$

by closedness of the symplectic form and nilpotency of exterior derivative $d$.

Basic hydrodynamical interpretation of canonical formalism will also be of some value. Let us consider phase-fluid of many systems with various initial conditions, moving on the phase-space [25]. Velocity field of the fluid is clearly given by Hamiltonian vector field. Note that since $H \in \mathcal{C}^{2}(D)$, we have $\operatorname{div} \boldsymbol{v}=0$.

It surely obeys the continuity law

$$
\frac{\partial \rho}{\partial t}+\operatorname{div}(\rho \boldsymbol{v})=0
$$

or in another words

$$
\frac{d \rho}{d t}+\rho(\nabla \cdot \boldsymbol{v})=0
$$

Now because the phase fluid is incompressible

$$
\rho=\text { const }
$$

on a target energy level set. Now let us ponder $\rho=C$ and then we vary the constant as $C=C(x, p)$ to get the condition

$$
\nabla C \cdot \boldsymbol{v}=0 \rightarrow C=c H
$$

where $c$ is a rightful constant.

Fluid should also undergo Euler's equation

$$
\frac{d v}{d t}=-\frac{1}{\rho} \nabla P
$$

which in elementary manner leads to Bernoulli's law

$$
\frac{1}{2} c\left(x^{2}+p^{2}\right)+P(\boldsymbol{x})=\text { const }
$$

from which we can evaluate the pressure function and constant appearing in the formula, as pressure needs to be non-negative. 


\section{$3 \mathcal{M}$-systems of ecology and chemical kinetics}

Hamiltonian (in general Poisson) structure can be met also in ecology ${ }^{1}$, where evolving populations of different species share resources in a common domain of living. Various environmental factors also can be modeled through some additional terms in differential equations governing the evolution (optimal control problems etc. [4])

We point out that similar approach can be adapted for chemical kinetics problems, where the role of populations is played by concentrations of various different chemical substances. Resource function here, if it exists, reflects amount of reacting substances.

In both domains the systems share properties of non-negativity, realizability, reducibility and semi-stability $[5,8,14]$.

Mentioned processes may involve great number of variables, if they are to be described exactly. For our purposes we will stick to ODE models, as fair enough to describe phenomena with satisfying accuracy, yet simple not to complicate things unnecessarily, so we use the assumptions

1. Reagents (species) are well-mixed (distributed homogeneously), otherwise the problem would be inhomogeneous in space, hence yielding PDE (reaction-diffusion problem, see e.g. [13]) instead of ODE.

2. Concentrations of substrates (species) are big enough to prevent stochastic behavior during reacting (coexisting - abandonment of this assumption would lead to Wiener processes). Otherwise we would get SDE instead of ODE.

Generally we could consider few types of interaction between species - parasitic invasion, competition for resources, etc. For example, competition of $U$ and $V$ given by the system

$$
\begin{aligned}
& \dot{u}=f(u, v), \\
& \dot{v}=g(u, v)
\end{aligned}
$$

would undergo conditions $f_{u}<0, g_{v}<0, f_{v}<0, g_{u}<0$ as to reflect fact that consumption of a nutrient/depletion of a resource by one species would prevent the other from doing the same, and also describing the competition between the members of the same population.

Specific kind of interaction appears in systems of Rosenzweig-MacArthur [6, 38] type, involving predator and prey coexistence. In (3.1) we would get

$$
\begin{aligned}
& f(u, v)=r u\left(1-\frac{u}{K}\right)-v h(u), \\
& g(u, v)=v(-\beta+\alpha h(u),
\end{aligned}
$$

where $h(u)$ denotes number of prey caught by predator per unit time and $\alpha, \beta, r, K$ are constant environmental parameters of the system.

Functions as $h(u)$ are known as functional response of predator to variation in prey population. Basic types of these were classified by C.S.Holling in three categories: I. Linear, II. Hyperbolic (saturation), III. So-called $\theta$-sigmoid [38].

Various parameters in the equations of population dynamics can be made into variable ones, often leading to their re-appearance as new dynamical (dependent) variables. To study these and more of not only single-population models like e.g. chemostat system with continuous/batch

\footnotetext{
${ }^{1}$ Sometimes the term "m-systems" is used to describe molecular systems in biology, however we stick to the meaning proposed in [28] as accepted by scientific community (e.g. [12]).
} 
cultivated bacteria, Monod equation, grazers-vegetation cycles, Ivlev or Ayala-Gilpin-Ehrenfeld model of population growth, epidemic and endemic (SIR/SIS) models, or even more complicated problems associated with optimal control of invasive species and harvesting of populations, the reader is referred to literature on the subject $[1,4,37]$.

Of course, some of these systems admit Hamiltonian/Poisson representation, where we will conceive of the resources function $\mathcal{M}(\boldsymbol{x}, t)$ as the basic object. It serves as a generator for equations of motion

$$
\dot{x}=B(\boldsymbol{x}, t) \nabla \mathcal{M}
$$

Remark 3.1. Similarly we can formulate Hamiltonian-like $\mathcal{M}$-system substituting $J$ in place of $B$ and properly transforming variables. However, big difference occurs when we ponder both Poisson and Hamilton formulations of the same problem: the concentration variables for different species should be of non-negative values, but in Hamiltonian case are not (incompressible fluid on $\mathbb{R}^{m}$ ). Because of that, we can interpret the compressibility of the phase fluid in the Poisson case as partially arising from constraining the phase-space to be $\mathbb{R}_{+}^{m}$.

Set of chemical reactions is termed reaction network. Having

$$
A_{i}+E_{i} \rightarrow P_{i}+B_{i}, \quad i=1, \ldots, n
$$

we call species on the left the reactants and species on the right products of the reaction.

When all stoichiometric coefficients are equal to one, we call such reaction an elementary reaction. Note that every particular reaction can be written as elementary reaction when we substitute $A_{1} X=X+\ldots+X$ ( $A_{1}$ times).

Chemical reactions formulated as populations dynamics problem use the mass action law [8, 37]: At constant temperature, for any elementary reaction, its rate is proportional to concentrations of reactants.

Matrix formulation is also accessible to problems concerning mass action law, see e.g. [5, 8]. This simple rule is underlying differential description of such reactions as given in the Michaelis-Menten model, Hill enzymatic equation, or Robertson network.

One of fundamental features of mass-action knetics is that it produces differential equations with polynomial non-linearities. This also means, that when we encounter such set of equations, we may find reaction network obeying these. Such process is referred to as realizibility of mass action kinetics [8]. Example of this procedure may be so-called Lotka-Volterra reactions, retrieved from $(5.8)$, see $[5,7]$.

\section{Effectively Hamiltonian description of non-conservative sys- tems}

We will proceed case-wise, slowly building efficient machinery, to achieve our goal as outlined in $[29,30]$.

We start with elementary example of a system

$$
\begin{aligned}
& \dot{x}=p, \\
& \dot{p}=-V^{\prime}(x)-D(x, p),
\end{aligned}
$$


where we need two pieces of dynamical structure on the phase space: potential function $V(x)$ (given only in terms of generalized coordinates) and $D(x, p)$ - non-potential force introducing energy flow out of and into the system

$$
\dot{H}=-D(x, p) p .
$$

Theory of ODEs confirms that if we are able to find unique solution to the problem, then phase trajectories do not intersect, in order to keep vector field generating the ODE well defined. This means, provided sufficiently differentiable $V$ and $D$, that any given instant $t$ in time connects by $1: 1$ correspondence to some $(x, p)$, and vice-versa.

We adjoin dissipative forces to the system, counting their work as positive. Then decrement of the energy of potential part of the system is exactly balanced by addition of the redundant variable

$$
w(t)=\int_{t_{0}}^{t} D\left(x\left(t^{\prime}\right), p\left(t^{\prime}\right)\right) d x\left(t^{\prime}\right)
$$

called a reservoir variable. In the above formula we perform the Riemann-Stieltjes integral guaranteed there exists some form of $1: 1$ correspondence between dynamical variables.

Theorem 4.1. The quantity

$$
K=H+w
$$

is conserved.

Proof. We may easily check

$$
\dot{K}=\dot{H}+\dot{w}=-D(x, p) p+D(x, p) p=0,
$$

however, it is not a well-defined function, since it depends on path taken by the system.

Above result is important not because of its complicated nature, but because of its simplicity. We accessed novel type of conserved quantity that is rather of no use in pure theoretical considerations. As outlined in the introduction, it is of huge practical/computational value.

Let us observe that $K$ possesses extremely trivial physical interpretation: it is just initial energy (provided that $\left.w\left(t_{0}\right)=0\right)$. Since, for general $\left(x_{0}, p_{0}\right), w\left(t_{0}\right)=$ const, we have $K=H\left(x_{0}, p_{0}\right)+w\left(t_{0}\right)$ and therefore $K$ is a smooth function of initial values provided that $H$ is smooth.

We call differential form not being the differential of any function a (pure) Pfaffian form [33]. Example of such quantity is

$$
đ w=D(x, p) d x
$$

where we understand its derivative of $w$ with respect to $x$ as $\frac{\partial w}{\partial x}:=\frac{1}{\dot{x}} \frac{d w}{d t}$, rather a differential quotient, than rightful derivative. In future, we will denote by $w_{x}$ expression standing next to $d x$ in $w$, the same for $w_{p}$.

Therefore we can define

$$
\varpi K=đ H+đ w
$$


as an improper (Pfaffian) differential form.

In the above definition we paid attention to the fact that Hamiltonian is no longer preserved. Its decrement is exactly the increment of $w$ with opposite sign. Since change of Hamiltonian now obviously depends on the path taken by the system on phase space, we cannot even claim that Hamiltonian is still potential-type function, or properly defined function at all, although, as a shortcoming, we will use the term "potential part of the generator of motion" with respect to Hamiltonian (so, strictly speaking, $d H$ is becoming Pfaffian form).

From earlier considerations we may generalize (2.4) to

$$
X_{K}=\pi(\cdot, d K)
$$

giving rise to Poisson bracket

$$
\{f, K\}=X_{K}(f),
$$

for any function given on the phase space. We can conceive of $K$ as the generator of non-potential motion: its Poisson bracket with canonical coordinates gives proper equations of motion

$$
\begin{aligned}
& \dot{q}=\{q, K\}=\pi(d q, K)=p, \\
& \dot{p}=\{p, K\}=\pi(d p, K)=-V^{\prime}(q)-D(q, p)
\end{aligned}
$$

moreover

$$
\dot{w}=\{w, K\}=\pi(d w, d K)=D(q, p) p,
$$

which is indeed the case.

With this setting in mind we can derive new formulas for vector field algebra. Considering $K$ generator of motion and $f$ well-differentiable function we obtain

$$
\left[X_{f}, X_{K}\right]_{L}=-X_{\{f, K\}}+\operatorname{div} \boldsymbol{v} X_{f},
$$

seeing that compressible terms which are responsible for discontinuities are causing an anomaly in the vector field algebra to occur.

It is possible to find similar formula for pair of reservoir-containing $K, L$, say. However, it is not very useful in the context of Hamiltonian description of mechanical systems since there is only one of these needed to govern dynamics. Situation dramatically changes in Nambu, or generalized Nambu mechanics (e.g. [32]), where dynamics is given in terms of few of such vector fields.

We will stick to this working approach, especially since it guarantees

$$
\omega=d x \wedge d p
$$

as a symplectic form. During former considerations it was preserved by the flow of Hamiltonian vector field $X_{H}$, now it satisfies

$$
\left.\left.\mathcal{L}_{X_{K}}(\omega)=X_{K}\right\rfloor d \omega+d\left(X_{K}\right\rfloor \omega\right)=d(d K)=d(d H+\varpi w)=d(-\succsim w+d w)=0 .
$$

$X_{K}$ is defined unambiguously throughout the phase-space as a section over $T\left(T^{*} M\right)$, hence providing phase trajectories not crossing each other. 
When it comes to the value of $K$, it is determined by providing initial conditions $\left(\boldsymbol{q}_{0}, \boldsymbol{p}_{0}\right)$. Hence $K \in \mathcal{F}\left(T^{*} M\left(t_{0}\right)\right)$, so its constant value is uniquely determined by the state of the system in initial moment. As a function of the flow $K$ may be given as

$$
\left.K=\int_{t_{0}}^{t} \boldsymbol{v}\right\rfloor \omega
$$

making its dependence on initial conditions much less manifest.

A little bit more sophisticated is simultaneous use of two reservoirs for the system

$$
\begin{aligned}
& \dot{x}=p+E(x, p), \\
& \dot{p}=-V^{\prime}(x)-D(x, p),
\end{aligned}
$$

yielding

$$
d K=(p+E(x, p)) d p+\left(V^{\prime}(x)+D(x, p)\right) d x
$$

and accordingly

$$
X_{K}=(p+E(x, p)) \frac{\partial}{\partial x}-\left(V^{\prime}(x)+D(x, p)\right) \frac{\partial}{\partial p} .
$$

Since we play with ODE system, when we assume that $A(\boldsymbol{x}) \nabla K=\boldsymbol{f}(\boldsymbol{x}) \in \mathcal{C}^{r}(D)$, $r \geq 1$, theorem on existence and uniqueness of solutions is in power $[2,19,21]$. Therefore there is a $1: 1$ correspondence between every moment in time, and points in phase-space. Trajectories on the phase space of the system are obviously not-crossing. Hence we can perform in an unambiguous sense any integral of a function of variables of the system with respect to some of these variables (or time) as a Riemann-Stieltjes integral.

Since that we can write

$$
K=\frac{1}{2} p^{2}+V(x)+w+z
$$

where the reservoirs are defined by

$$
\begin{aligned}
& w=\int_{t_{0}}^{t} D\left(x\left(t^{\prime}\right), p\left(t^{\prime}\right)\right) d x\left(t^{\prime}\right), \\
& z=\int_{t_{0}}^{t_{0}} E\left(x\left(t^{\prime}\right), p\left(t^{\prime}\right)\right) d p\left(t^{\prime}\right),
\end{aligned}
$$

so that $\dot{K}=0$.

Now, in order to make current discussion as similar to the conservative case as possible, we focus for a moment on hydrodynamical analogy, starting from continuity equation

$$
\frac{d \rho}{d t}+\rho \nabla \cdot \boldsymbol{v}=0
$$

hence

$$
\rho=C e^{\int_{t_{0}}^{t} D_{p}(q, p) d t^{\prime}}
$$

where $C$ is a constant and lower index denote derivative with respect to an argument. 
Integral in the exponent does not cause any trouble, since all fluid's particles obey equations of motion, hence we may again apply the Riemann-Stieltjes integral.

Note that we can consider $C$ as depending on canonical variables, where from continuity equation we get the constraint $\boldsymbol{v} \cdot \nabla C$, so $C$ may depend on $K$ value, so on the phase trajectory of interest.

Additionally we have Bernoulli's law (from Euler's equation)

$$
\frac{1}{2} \rho \boldsymbol{v}^{2}+P=\text { const. }
$$

Taking an example of linearly damped harmonic oscillators with equation of motion

$$
\begin{aligned}
& \dot{x}=p, \\
& \dot{p}=-x-b p .
\end{aligned}
$$

Since all fluid particles obey these equations of motion, continuity equation yields

$$
\rho=c K e^{b t},
$$

where $c$ is a constant.

Writing Bernoulli's law

$$
\frac{1}{2} c K e^{b t}\left(x^{2}+p^{2}+2 b p x+b^{2} p^{2}\right)+P=\text { const },
$$

and remembering that $E \sim e^{-b t}$ we see

$$
P=P_{0}-c K e^{b t}\left(b x p+\frac{1}{2} b^{2} p^{2}\right) .
$$

Fortunately we know solutions to the damped oscillator, being $x \sim e^{-\frac{b}{2} t}(\cos (\omega t+\delta))$, hence $p \sim e^{-\frac{b}{2} t}(\cos (\omega t+\delta)-\omega \sin (\omega t+\delta))$. Thus we see there is no danger of the variable part of pressure growing to infinity. Provided that engaged constants obey

$$
P_{0}-c K A_{0}^{2}\left[\left(b+\frac{b^{2}}{2}\right) \cos ^{2} \delta-\left(\omega b+b^{2}\right) \sin \delta \cos \delta+\frac{1}{2} b^{2} \omega^{2} \sin ^{2} \delta\right] \geq 0,
$$

where $A_{0}$ is initial amplitude, pressure is always positive. Notice that for different $K$ (initial energy) this demand can somewhat change quantitatively.

\section{Particular non-potential systems}

Here we place a sequence of illustrative examples, treatable along the lines of presented approach. Their objective is to show applicability of the invented framework to low-dimensional systems. As mentioned in an introduction, general statement with the proof will be published elsewhere.

\section{Example 5.1. Van der Pol oscillator}

Van der Pol oscillator is a system that arises as some generalization of a RLC circuit (through so-called Liénard form equation of VdP oscillator [19]) and its equations of motion are

$$
\begin{aligned}
& \dot{x}=p, \\
& \dot{p}=-x+\varepsilon\left(1-x^{2}\right) p .
\end{aligned}
$$


Non-potential generator of motion is

$$
K=\frac{1}{2}\left(x^{2}+p^{2}\right)+w,
$$

where the reservoir variable is given by

$$
w=\varepsilon \int_{t_{0}}^{t}\left(1-x\left(t^{\prime}\right)^{2}\right) p\left(t^{\prime}\right) d x\left(t^{\prime}\right),
$$

turning $K$ into effectively conserved quantity.

\section{Example 5.2. Brusselator}

Brusselator is the dynamical system modeling auto-catalytic reaction network [26]

$$
A \rightarrow X, \quad 2 X+Y \rightarrow 3 X, \quad B+X \rightarrow Y+D, \quad X \rightarrow E .
$$

We claim that substrates $A, B$ are abundant in the environment, so we can denote their concentrations $a, b$ as being constant. We treat $X$ and $Y$ species concentrations as dynamical variables. From (5.4), with the use of mass-action principle, we get

$$
\begin{aligned}
& \dot{x}=a+x^{2} y-b x-x, \\
& \dot{y}=b x-x^{2} y .
\end{aligned}
$$

Short look at these confirms there is no resource function $\mathcal{M}$ in the usual sense; the non-potential generator of motion becomes

$$
K=a y-\frac{1}{2} x^{2} y+w+z,
$$

where the reservoir variables are given by

$$
\begin{aligned}
& w=\int_{t_{0}}^{t} x\left(t^{\prime}\right)^{2} y\left(t^{\prime}\right) d x\left(t^{\prime}\right), \\
& z=\int_{t_{0}}^{t}\left(x^{2}\left(t^{\prime}\right) y\left(t^{\prime}\right)-b x\left(t^{\prime}\right)-x\left(t^{\prime}\right)\right) d y\left(t^{\prime}\right),
\end{aligned}
$$

so that $\dot{K}=0$.

Note that (5.5) has fixed point at $\left(a, \frac{b}{a}\right)$. This equilibrium is unstable when $b>1+a^{2}$, if $b<1+a^{2}$ it is stable. Case $b=1+a^{2}$ presents some doubts: in this situation, the origin appears to be the center (from the procedure of linearization), however we know that if the dimension (here: number of dependent variables) of ODE system $n \geq 2$, then Hartman-Grobman theorem on linearization often fails at predicting existence of the centre [19, 21, ?].

\section{Example 5.3. Lotka-Volterra system}

Lotka-Volterra model describes basic predator-prey interaction (with linear response)

$$
\begin{aligned}
& \dot{u}=u-\alpha u v \\
& \dot{v}=-\beta v+u v
\end{aligned}
$$

where $u$ is prey concentration in an environment, $v$ is predator concentration, $\alpha$ being the rate at which consumption af prey by a predator proceeds, and $\beta$ is the death rate of a predator. Note that we choose unit rate for birth of prey and predator feed on prey. 
We can write down these equations as a Poisson system

$$
\dot{\boldsymbol{x}}=B(\boldsymbol{x}) \nabla \mathcal{M}, \quad B(\boldsymbol{x})=\left(\begin{array}{cl}
0 & u v \\
-u v & 0
\end{array}\right)
$$

claiming that $\boldsymbol{x}=(u, v)^{T}, \nabla=\left(\partial_{u}, \partial_{v}\right)^{T}$ and the resource function is

$$
\mathcal{M}=\beta \ln u+\ln v-u-\alpha v .
$$

We should observe that such formulated LV problem is given on the phase space $\mathbb{R}_{+}^{2}$, and as a Poisson system has compressible phase fluid: div $\dot{\boldsymbol{x}}=1-\beta-\alpha v+u$ con. rem. (3.1).

We use that the Poisson structure as even-dimensional and non-degenerate, so we can bring the system to its canonical form by transformation $u=e^{q}, v=e^{p}$, where equations of motion becomes

$$
\begin{aligned}
& \dot{q}=1-\alpha e^{p} \\
& \dot{p}=-\beta+e^{q}
\end{aligned}
$$

with incompressible phase-fluid on $\mathbb{R}^{2}$ symplectic phase-space with separable resorce function $\mathcal{M}=$ $p-\alpha e^{p}+\beta q-e^{q}$.

Note that LV Poisson system would become of canonical form also with

$$
d K=u(1-\alpha v) d v+v(\beta-u) d u
$$

Moreover we have

$$
\nabla K^{T} B(\boldsymbol{x}) \nabla \mathcal{M}=0=\nabla \mathcal{M}^{T} J \nabla K
$$

hence integrals of motion are commuting in terms of each Poisson structure, but this only preserves equilibria. What is more important we have

Corollary 5.4. Transition from Poisson dynamics governed by the resource function $\mathcal{M}$ in (5.10) to canonical form evolution of which is dictated by (5.12) preserves Poisson bracket of a target function with generator of motion (with its coupled matrix structure).

This remark is easily verifiable on case-to-case basis, provided that the Poisson bracket of $\mathcal{M}$ with coordinates $u, v$ is preserved.

Knowing that non-potential generator $K$ provokes anomalies of the vector fields to occur, we expect, and then obtain

$$
\left[X_{f}, X_{K}\right]_{L}=-X_{\{f, K\}}+\operatorname{div}\left(X_{K}\right) X_{f}
$$

\section{Example 5.5. Robertson reactions}

In this example and afterwards, we omit t' argument explicitly appearing in all reservoir integrals. Reaction network

$$
X \stackrel{a}{\rightarrow} Y, \quad Y+Y \stackrel{b}{\rightarrow} Y+Z, \quad Y+Z \stackrel{c}{\rightarrow} X+Z
$$

is a system of auto-catalytic reactions where $a, b, c$ are reaction rates. 
Mass-action law gives system clearly expressible in gradient form $\left(\boldsymbol{x}=(x, y, z)^{T}\right)$

$$
\dot{\boldsymbol{x}}=B(\boldsymbol{x}) \nabla H, \quad B(\boldsymbol{x})=\left(\begin{array}{ccc}
0 & c y z+b y^{2} & -a x-b y^{2} \\
-c y z-b y^{2} & 0 & a x \\
a x+b y^{2} & -a x & 0
\end{array}\right)
$$

with conserved $H=x+y+z$ (classical rule of mass conservation). Note additionally, that $B(\boldsymbol{x})$ does not obey Jacobi's identity, although its skew-symmetry itself guarantees conservation property [29, 34].

We are able to write down the system in different form

$$
\dot{x}=\varepsilon \nabla K
$$

$\varepsilon$ being totally anti-symmetric Cartesian-tensor of order 3 and

$$
K=-\frac{1}{2} a x^{2}-\frac{1}{3} b y^{3}-a \int_{t_{0}}^{t} x d y-\int_{t_{0}}^{t}\left(b y^{2}+c y z\right) d z
$$

therefore we need a pair of reservoirs. In this form system is Poisson one: anti-symmetric structure matrix obeys Jabobi identity, moreover, the system admits Casimir function H, since it is obvious that $\varepsilon \nabla H=0$, hence we can proceed the construction of Darboux coordinates on a single symplectic leaf of the system, e.g. $(y, z)$, where $x=m_{0}-y-z, m_{0}$ constant.

System reduces to

$$
\begin{aligned}
& \dot{y}=\mu-a y-a z-b y^{2}-c y z=-K_{z}, \\
& \dot{z}=b y^{2}=K_{y},
\end{aligned}
$$

where $\mu=a m_{0}$. To cast above system in gradient form we need only single reservoir

$$
K=-\mu z+\frac{1}{2} a z^{2}+\frac{1}{3} b y^{3}+\int_{t_{0}}^{t}\left(a y+b y^{2}+c y z\right) d z
$$

and it is explicitly of canonical form.

It is worth stressing that we can apply Casimir function to generator governing evolution of the system (5.18) to reduce number of variables, but the formula will be different from that obtained applying given Casimir to equations of motion, and then finding the reduced generator (5.20). Results are obviously unequal, but their differentials are cohomologically equivalent [2].

Example 5.6. To illustrate that ideas presented here work also in higher dimensions, we ponder on five-dimensional system considered in [17].

$$
\begin{aligned}
& \dot{p}=\alpha(z)-\beta p y+\gamma p q, \\
& \dot{q}=\delta(z) q-\varepsilon q x-\gamma p q, \\
& \dot{x}=\varepsilon q x-\zeta x, \\
& \dot{y}=\beta p y-\eta y, \\
& \dot{z}=-\alpha(z) p-\delta(z) q+\eta y+\zeta x .
\end{aligned}
$$

Summing all the equations together we find

$$
\frac{d}{t}(p+q+x+y+z)=\alpha(z)(1-p)
$$


sand performing the Riemann-Stieltjes integral, we gain access to

$$
p+q+x+y+z-\int \alpha(z)(1-p)=\mathrm{const}=C_{0}
$$

on target level set.

Eliminating $x$ we obtain

$$
\begin{aligned}
& \dot{p}=\alpha(z)-\beta p y+\gamma p q \\
& \dot{q}=\delta(z) q-\varepsilon q\left(C_{0}+\int \alpha(z)(1-p) d t-p-q-y-z\right)-\gamma p q \\
& \dot{y}=\beta p y-\eta y \\
& \dot{z}=-\alpha(z) p-\delta(z) q+\eta y+\zeta\left(C_{0}+\int \alpha(z)(1-p) d t-p-q-y-z\right) .
\end{aligned}
$$

which is writable in Hamiltonian form with four reservoirs, provided we choose canonical pairs, for example $(p, y),(q, z)$.

Observe interesting novelty: traditional first integrals led to reduction of order of ODE by one; effectively conserved quantities turn the problem into integro-differential one instead. Meaning of this fact and our capability to cope with it numerically will be discussed in future papers.

\section{The proposition and conclusions}

Leaning against the results of $[16,22,29,34]$ and research done in former sections of this paper, we can propose new numerical ethod in discrete gradient family of algorithms.

We stipulate that we work far from any degenerated fixed point of the system, since it would couse numerical approximation of $B$ not to be locally bounded [29].

Our new algorithm in case of one-dimensional system is

$$
\begin{aligned}
& \frac{x^{\prime}-x}{h}=\frac{K\left(x^{\prime}, p^{\prime}, w^{\prime}\right)-K\left(x^{\prime}, p, w^{\prime}\right)}{p^{\prime}-p} \\
& \frac{p^{\prime}-p}{h}=-\frac{K\left(x^{\prime}, p, w^{\prime}\right)-K(x, p, w)}{x^{\prime}-x} \\
& w^{\prime}=w+D\left(x^{*}, p^{*}\right)\left(x^{\prime}-x\right)
\end{aligned}
$$

and cross-multiplying first two of above equations, we see instantly it preserves $K$ exactly. Note the presence of additional third equation. It helps to sustain this property since the system alone is not conservative.

Provided simple assumptions

$$
\begin{aligned}
& \nabla K\left(x, p, x^{\prime}, p^{\prime}\right) \cdot\left(\boldsymbol{x}^{\prime}-\boldsymbol{x}\right)=K\left(x^{\prime}, p^{\prime}\right)-K(x, p), \\
& \bar{\nabla} K(\boldsymbol{x}, \boldsymbol{x})=\nabla K(\boldsymbol{x})
\end{aligned}
$$

we get consistent scheme.

As an example let us consider Duffing oscillator with damping

$$
\begin{aligned}
& \frac{x^{\prime}-x}{h}=\frac{K\left(x^{\prime}, p^{\prime}, w^{\prime}\right)-K\left(x^{\prime}, p, w^{\prime}\right)}{p^{\prime}-p} \\
& \frac{p^{\prime}-p}{h}=-\frac{K\left(x^{\prime}, p, w^{\prime}\right)-K(x, p, w)}{x^{\prime}-x} \\
& w^{\prime}-w=b p^{*}\left(x^{\prime}-x\right)
\end{aligned}
$$


where $b$ is damping constant; this becomes

$$
\begin{aligned}
& \frac{x^{\prime}-x}{h}=\frac{1}{2}\left(p+p^{\prime}\right) \\
& \frac{p^{\prime}-p}{h}=\frac{1}{2}\left(x+x^{\prime}\right)-\frac{1}{4}\left(x^{3}+x^{2} x^{\prime}+x x^{\prime 2}+x^{\prime 3}\right)-\frac{b}{2}\left(p+p^{\prime}\right), \\
& w^{\prime}-w=\frac{b}{2}\left(p+p^{\prime}\right)\left(x^{\prime}-x\right)
\end{aligned}
$$

where we have chosen $p^{*}=\frac{1}{2}\left(p+p^{\prime}\right.$ ) (but it can be chosen otherwise, we explore this freedom in a future paper), since the only condition needed to be obeyed is $\lim _{h \rightarrow 0} p^{*}=p$. Important remark concerning application of the method is that we modify reservoir variable $w$ at the same time we do so for its integration variable $x$.

To sum up, we obtained new kind of conserved quantity, for distinction called effectively conserved quantity. Its existence is not a consequence of equations of motion alone; it is preserved due to equations of motion after adjoining reservoir(s) to the system.

Constant quantities have reduced order of differential equation, each of them by one. Effectively conserved quantities can only turn differential equations into integro-differential ones, hence they are not of huge analytical advantage, although they can help us to reduce dynamical system to canonical form, as in the example (5.6).

Additionally, it is clearly implied that every non-degenerate Poisson system (even a non-potential one!) admits canonical representation in which effectively conserved quantity can be perceived as a generator of motion. With this under our belt, we can head towards multiple applications of the idea of this paper in computational techniques for ODEs; for example we can construct gradient integrators for non-potential systems. This is a huge novelty, since up till now, this class of systems in general refused the treatment (minus systems with Lyapunov function). The result yields hopes connected not only with presented framework, but also parallel approach to non-autonomous systems being constructed and multi-gradient systems being of central interest in Nambu mechanics [32].

Acknowledgment:

Author is very grateful to Prof. J.L.Cieśliński for encouragement and multitude of discussions leading to origination of this text.

\section{References}

[1] M.Al-Moqbali, N.Al-Salti, I.Elmojtaba: Prey-Predator Models with Variable Carrying Capacity, Mathematics 6 (2018), MDPI, DOI: 10.3390/math6060102

[2] V.I.Arnold: Mathematical methods of classical mechanics, Springer-Verlag, New York, 1978,

[3] O.Babelon, D.Bernard, M.Talon: Introduction to Classical Integrable Systems, Cambridge Monographs on Mathematical Physics, DOI 10.1017/CBO9780511535024, Cambridge University Press, 2003,

[4] C.M.Baker, F.Deile, D.Lacitignola, C.Marangi, A.Martiradonna: Optimal control of invasive species through a dynamical systems approach, Nonlinear Analysis: Real World Applications (49), Elsevier 2019,

[5] E.Bertolazzi: Positive and conservative schemes for mass action kinetics, Computers Math. Applic. Vol. 32 No. 6, 1996, Pergmon,

[6] F.Brauer,C.Castillo-Chavez: Mathematical Models in Population Biology and Epidemiology, Springer-Verlag, New York 2012,

[7] B.Boros, J.Hofbauer, S.Müller et al.: The Center Problem for the Lotka Reactions with Generalized MassAction Kinetics, Qual. Theory Dyn. Syst. 17, 403 âĂŞ410 (2018). https://doi.org/10.1007/s12346-017-0243-2

[8] V.Chellaboina, S.Bhat, W.Haddad, D.Bernstein: Modeling and analysis of mass-action kinetics, Control Systems, IEEE 29 (2009), DOI: 10.1109/MCS.2009.932926,

[9] J.L.Cieśliński: Locally exact modifications of numerical schemes, Comput. Math. Appl. 65(2013),

[10] J.L.Cieśliński, A.Kobus: Locally Exact Integrators for the Duffing Equation, Mathematics, 8, 231, MDPI 2020, 
[11] J.L.Cieśliński, B.Ratkiewicz: Long-time behavior of discretizations of the simple pendulum equation, J.Phys. A: Math. Theor. 42 (2009), 29 pp.

[12] F.Diele, C.Marangi: Geometric Numerical Integration in Ecological Modeling, DOI: 10.3390/math8010025, Mathematics, MDPI 202

[13] F.Diele, C.Marangi, S.Ragni: IMSP schemes for spatially explicit models of cyclic populations and metapopulation dynamics, Mathematics and Computers in Simulation (110), Elseevier 2015,

[14] L.Formaggia, A.Scotti, Positivity and Conservation Properties of Some Integration Schemes for Mass Action Kinetics, SIAM J. Numerical Analysis 49(2011), DOI: 10.2307/23074332,

[15] L.C.García-Naranjo, J.C.Marrero: The geometry of nonholonomic Chaplygin systems revisited, 2020 IOP Publishing Ltd \& London Mathematical Society, Nonlinearity, Volume 33, Number 3,

[16] Gonzalez

[17] S.A.Hadley, L.K.Forbes: Dynamical Systems Analysis of a Five-Dimensional Trophic Food Web Model in the Southern Oceans, Journal of Applied Mathematics Volume 2009, Article ID 575047, 17 pages, doi:10.1155/2009/575047, Hindawi Publishing Corporation,

[18] E.Hairer, C.Lubich, G.Wanner: Geometric numerical integration: Structure preserving algorithms for ordinary differential equations, Springer Verlag 2006,

[19] J.K.Hale, H.Kocak: Dynamics and bifurcations, Springer New York, 1996

[20] T.Honein, N.Chien, G.Herrmann: On conservation laws for dissipative systems, Physics Letters A, Volume 155, Issues 4âĂŞ5, 13 May 1991, Pages 223-224,

[21] M.W.Hirsch, S.Smale: Differential Equations, Dynamical Systems, and Linear Algebra, Academic Press. INC. San Diego, California, 1974,

[22] T.Itoh, K.Abe: Hamiltonian-conserving discrete canonical equations based on variational difference quotients, Journal of Computational Physics vol. 76 (1), Elsevier 1988,

[23] B.Karasözen: Poisson integrators, Mathematical and Computer Modelling, Volume 40, Issues 11ї£¡12, 2004 Elsevier, Pages 1225-1244,

[24] Kovacic, Brennan

[25] V.V.Kozlov: Hamiltonian Systems with Three Degrees of Freedom and Hydrodynamics in Hamiltonian Systems with Three or More Degrees of Freedom, ed. C.SimÃş, Springer-Verlag, Part of the NATO ASI Series book series (ASIC, volume 533),

[26] Y.A.Kuznetsov: Elements of Applied Bifurcation Theory, Springer-Verlag New York, 1995,

[27] M.León, C.Sardón: A geometric approach to solve time dependent and dissipative Hamiltonian systems, arXiv: 1607.01239v1 [math-ph], 2016,

[28] J.Martinez-Linares: Phase Space Formulation of Population Dynamics in Ecology, arXiv: 1304.2324 [q-bio],

[29] R.I.McLachlan, G.R.W.Quispel, N.Rubidoux: Geometric integration using discrete gradients, Phil. Trans.R. Soc.Lnd. A, 357(1999),1021-1045.

[30] R.Mclachlan, G.Quispel, N.Robidoux, Unified Approach to Hamiltonian Systems, Poisson Systems, Gradient Systems, and Systems with Lyapunov Functions or First Integrals, DOI: 10.1103/PhysRevLett.81.2399, Physical Review Letters vol. 81, 1998,

[31] R.E.Mickens: Numerical integration of population models satisfying conservation laws: NSFD methods, J Biol Dyn. 2007;1(4):427-436. doi:10.1080/17513750701605598,

[32] Y.Nambu: Generalized Hamiltonian Dynamics, Phys. Rev. D 7(8), 1973, American Physical Society,

[33] P.Popescu, M.Popescu: On Pfaff systems, BSG Proceedings, 2012

[34] G.R.W.Quispel, H.W.Capel: Solving ODEs mumerically while preserving a first integral, Elsevier, Physics Letters A, 218(1996), 223-228.

[35] F.Schwarz, W-H.Steeb: Symmetries and first integrals for dissipative systems, Journal of Physics A: Mathematical and General, Volume 17, Number 15, IOP 1984,

[36] E.Süli, D.F.Mayers: An Introduction to Numerical Analysis, Cambridge University Press, Cambridge 2003, DOI 10.1017/CBO9780511801181

[37] S.H.Strogatz: Nonlinear Dynamics and Chaos: Nonlinear dynamics and chaos: with applications to physics, biology, chemistry, and engineering, Westview Press, a member of the Perseus Books Group, 2015

[38] P.Turchin: Complex population dynamics, Princeton University Press, 2003,

[39] A.Weinstein: The local structure of Poisson manifolds, J.Diff.Geom. 18 (1983), 523-557,

[40] A.Weinstein: Poisson geometry, Differential geometry and its applications, 9 (1998), 213-238, 